\title{
Effect of Quadriceps Muscle Strengthening Exercise on Quadriceps and Hamstring Muscle Strength Ratio in Patients with Osteoarthritis Grade 2 and 3
}

\author{
Thomas Sintong Pratama Purba, Marina Annette Moeliono, Sunaryo Barki Sastradimadja
}

Department of Physical Medicine and Rehabilitation, Faculty of Medicine, Universitas Padjadjaran-Dr. Hasan Sadikin General Hospital, Bandung

\begin{abstract}
Objective: To evaluate the effect of quadriceps muscle strengthening exercise on quadriceps and hamstring strength ratio in knee osteoarthritis (OA).

Methods: This was a quantitative study with quasi-experimental design. The subjects in the study were 16 female patients with knee OA grade 2 and 3 . Quadriceps muscle strengthening exercise was given to the subjects 3 times a week during 8 weeks. The study was conducted at the Department of Physical Medicine and Rehabilitation of the Faculty of Medicine, Universitas Padjadjaran-Dr. Hasan Sadikin General Hospital in August-September 2015.

Results: It was revealed that the quadriceps muscles improved significantly. However, the hamstring muscles did not show significant improvement based on the comparison between before and after performing the exercise. The average right quadriceps muscle strength before exercise was 12.46 and after exercise was 18. The average left quadriceps muscle strength before and after exercise were 11.59 and 17.7 , respectively. The average right hamstring muscle strength before and after exercise were 7.29 and 7.94 , respectively.

Conclusions: There are quadriceps muscle strength improvements after practicing quadriceps muscle strengthening exercise. However, no significant Received:

May 5, 2017 hamstring muscle strength improvement is seen after practicing quadriceps muscle strengthening exercise. An improvement in quadriceps and hamstring muscle ratio is observed.
\end{abstract}

Revised:

Keywords: Osteoarthritis, quadriceps and hamstring muscle strength ratio, Accepted: quadriceps muscle strengthening exercise

pISSN: 2302-1381; eISSN: 2338-4506; http://doi.org/10.15850/ijihs.v5n2.1041 IJIHS. 2017;5(2):64-9

\section{Introduction}

Knee osteoarthritis $(\mathrm{OA})$ is a joint disease that is commonly found in elderly. Knee OA affects $>40 \%$ Europeans and Americans, leading to disabilities. ${ }^{1}$ Osteoarthritis is a disease that is characterized by a joint cartilage degradation followed by the degradation in the subchondral bone and synovial joint capsule. ${ }^{2}$ Some of the $\mathrm{OA}$ factors are associated with the presence of

\footnotetext{
Correspondence:

Thomas Sintong Pratama Purba, Department of Physical Medicine and Rehabilitation, Faculty of Medicine, Universitas Padjajaran-Dr. Hasan Sadikin General Hospital

Jl. Pasteur No. 38, Bandung, Indonesia

e-mail: thomas.purba@gmail.com
}

mechanical loading and degenerative process. The clincal simptoms that are frequently found in $\mathrm{OA}$ patients include joint stiffness, joint range of motion (ROM) reduction, quadriceps muscle weakness, and proprioception internal changes. ${ }^{3}$

Knee as the largest joints providing support for the body weight is frequently affected by $\mathrm{OA}$. Knee OA patients find difficulties to do daily activities, especially those requiring mobilization as well as transference processes. Muscle strengths plays a role in the OA knee pathogenesis. Previous studies reported the correlation between muscle imbalance and muscle weakness in knee joints with several symptoms, e.g. decreases in functional ability, 
the presence of pains, and fall risks. ${ }^{2-5}$

Quadriceps and hamstrings are two largest muscles in the frontal plane. Co-contraction from the quadriceps and hamstring muscles can increase joint compressive forces, leading to knee joint stabilization. Dynamic stability in the frontal plane occurs due to quadriceps and hamstring abduction/aduction moment arm. Correlation between quadriceps and hamstring muscle strengths have been investigated in several studies. A study by Adogoke et $a l^{6}{ }^{6}$ described a lower quadriceps and hamstring muscle strength ratio in $\mathrm{OA}$ patients when compared to the healthy young population. Another study described a correlation between quadriceps and hamstring muscle strength ratio and symptomatic knee OA after practicing typical quadriceps and hamstring muscle strengthening exercises. ${ }^{2}$ In such, quadriceps muscle weakness was stated as a primary cause to quadriceps and hamstring value changes in OA knee patients. ${ }^{6}$

Several existing studies have reported that the hamstring muscles considerably influence the quadriceps and hamstring muscle strength rasio. Strengthening exercise is applied to knee OA patients based on findings that quadriceps muscle weakness is regarded as a major factor in the deviation of quadriceps and hamstring ratio value. ${ }^{3-5}$

The muscle strengthening exercise that is recommended to knee $\mathrm{OA}$ patients includes quadriceps muscle strengthening exercise with no weight bearing. This exercise program can be performed because it can distinctively strengthen the specific muscles and is appropriate for patients who experience pain when practicing weight bearing exercise. ${ }^{7}$ Therefore, this study aimed to analyze the effect of quadriceps muscle strengthening exercise to the quadriceps and hamstring muscle strength ratio.

\section{Methods}

Population in this study comprised of the knee OA patients who visited Physical Medicine and Rehabilitation clinic, Dr. Hasan Sadikin General Hospital, Bandung. The study was conducted in the period of August-September 2015. The subjects were members of the population who met the inclusion criteria and did not meet the exclusion criteria and were willing to participate in the study by signing the informed consent.

The number of subjects was defined using consequtive sampling approach; thus, the knee OA patients who met the criteria were included in the study for a certain period until the total required samples were obtained. The total required sample size was calculated based on a previous study with 16 subjects as the minimal numbers. This study used the pre-post designs with 1 group consisted of 16 subjects. The subjects were then given information related to the examination and intervention procedures. Participants who did not attend the exercise three times in a row are considered dropped out.

Inclusion criteria in this study were females aged 40-70 years, diagnosed with OA knee grade 2 and 3 in sub acute and chronic phases based on American College of Rheumatologi (ACR) and Kellgren Lawrence criteria, neutral alignment of knee joint, and able to walk with or without any gait aid. The exclusion criteria in the study were valgus-varus or recurvatum deformities, knee instabilities due to ligamentous laxity in cruciate ligament or collateral and meniscus injuries, stroke or certain neural system disorders, knee injury (fracture, ligament rupture or meniscus) or knee surgery, suffering from degenerative or metabolic diseases (arteriosclerosis, diabetes mellitus) or other inflammatory disorders, performing high intensity exercise (tennis, badminton) or attending other strengthening exercises in the last 6 months, and a numeric rating scale $(\mathrm{NRS})>6$.

The research method used in the study was quantitative with quasi-experimental design while the study design was experimental with one group pretest-posttest design. Data were calculated to analyze the effect of the treatment by using paired $t$ test for normally distributed data or Wilcoxon matched pairs test for nonnormally distributed data.

The study analyzed the correlation between dependent and independent variables. The independent variable was quadriceps muscle strengthening exercise while the dependent variables were quadriceps muscle strength, hamstring muscle strength, and quadriceps and hamstring muscle strength ratio. The confounding variables were body mass index (BMI) and age.

The subjects involved in the study were knee OA grade 2-3 patients given quadriceps muscle strengthening exercise. Comparative examination between the quadriceps and hamstring muscles were performed before and after the intervention. Subjects were asked to do quadriceps strengthening exercise for 8 weeks, 3 times a week. The exercise was conducted at the Department of Physical 
Table 1 Subject Basic Characteristics

\begin{tabular}{lcccc}
\hline \multicolumn{1}{c}{ Characteristics } & Mean/Median & Minimun & Maximum & SD \\
\hline Age & 57.875 & 40 & 70 & 10.7633 \\
Height & 1.525 & 1.47 & 1.61 & 0.04661 \\
Weight & 62 & 37 & 74 & 7.96633 \\
BMI & 26.399 & 16.015 & 31.203 & 3.241 \\
\hline
\end{tabular}

Note $\mathrm{SD}=$ standard deviation, $\mathrm{BMI}=$ body mass index

Medicine and Rehabilitation of the Faculty of Medicine, Universitas Padjadjaran-Dr. Hasan Sadikin General Hospital. The quadriceps and hamstring muscle strength examinations were performed before and after the study to investigate the muscle comparison after the exercise. This study used several devices for clinical examinations, such as height and weight measuring instruments, blood pressure monitor device, push-pull dynamometer, and quadriceps exercise bench.

Quadriceps strengthening muscle exercise is regarded as the most suitable excercise for $\mathrm{OA}$ patients. The exercise can be done dynamically and isotonically due to the fact that quadriceps muscles contract concentrically while the hamstring muscles will contract eccentrically as quadriceps muscle co-contractor. As such, quadriceps muscle strengthening exercise can improve hamstring muscles (Fig. 1). ${ }^{6}$

Quadriceps muscle strengthening exercise is a resistance exercise with external torque resulted by gravitation through the body. When the knee extension tibia on femoral, lower extremities external moment arm increase from $90^{\circ}$ to $0^{\circ}$ when knee flexion. In contrary, when knee extension femoral on tibia, external moment arm upper body weight decreased $90^{\circ}$ to $0^{\circ}$ when knee flexion (Fig. 2)..$^{2,6}$

The protocol applied for quadriceps muscle measurement was as follow: (a) the examiner held a dynameter with two hands, (b) the dynamometer was placed on the distal end of the tibia, (c) a muscle strength measurement were performed three time to obtain the average value as the measurement result.

The subjects in the study should performed the exercise protocols i.e. (1) doing warmingup and stretching exercise in 10 minutes before starting the quadriceps muscle strengthening exercise, (2) sitting on quadriceps exercise bench with stabilization, (3) given an exercise load based on 1 repetition maximum (RM) measurement performed every week at $60 \%$ intensity of $1 \mathrm{RM}$, (4) performing $90^{\circ} \mathrm{knee}$ extension against the load during the full knee extension in 2 seconds and going back to the starting position with flexible movement, (5) doing 3 set of 10 repetition exercise, (6) performing cooling down and streching in

Table 2 Correlation between Confounding Variable and Quadriceps and Hamstring Muscle Strengths

\begin{tabular}{lcccccccc} 
& RQBE & RQAE & LQBE & LQAE & RHBE & RHAE & LHBE & LHAE \\
\hline Age & -0.31 & -0.56 & -0.19 & -0.44 & -0.24 & -0.29 & -0.16 & -0.176 \\
& $\mathrm{p}=0.23$ & $\mathrm{p}=0.06$ & $\mathrm{p}=0.47$ & $\mathrm{p}=0.09$ & $\mathrm{p}=0.38$ & $\mathrm{p}=0.26$ & $\mathrm{p}=0.56$ & $\mathrm{p}=0.09$ \\
BMI & 0.15 & -0.18 & 0.33 & 0.05 & -0.21 & -0.18 & -0.08 & -0.18 \\
& $\mathrm{p}=0.58$ & $\mathrm{p}=0.49$ & $\mathrm{p}=0.2$ & $\mathrm{p}=0.84$ & $\mathrm{p}=0.44$ & $\mathrm{p}=0.5$ & $\mathrm{p}=0.77$ & $\mathrm{p}=0.51$ \\
\hline
\end{tabular}

Note: BMI= body mass index; LHAE= left hamstring after exercise; LHBE= left hamstring before exercise; LQAE $=$ left quadriceps after exercise; LQBE= left quadriceps before exercise; RHAE= right hamstring after exercise; RHBE $=$ right hamstring before exercise; $\mathrm{RQBE}=$ right quadriceps before exercise; $\mathrm{RQAE}=$ right quadriceps after exercise 
Table 3 Comparison between Quadriceps and Hamstring Muscle Strength in Before and After Performing Quadriceps Muscle Strengthening Exercise

\begin{tabular}{lccc}
\hline & $\begin{array}{c}\text { Mean Before } \\
\text { Exercise }\end{array}$ & $\begin{array}{c}\text { Mean After } \\
\text { Exercise }\end{array}$ & p Value \\
\hline Right quadriceps muscle strength & 12.46 & 18 & 0.00 \\
Left quadriceps muscle strength & 11.59 & 17.7 & 0.00 \\
Right hamstring muscle strength & 7.29 & 7.94 & 0.19 \\
Left hamstring muscle strength & 7.5 & 7.5 & 0.11 \\
\hline
\end{tabular}

10 minutes after the exercise. This study was approved by the Ethic Committee, Dr. Hasan Sadikin General Hospital, Bandung, Indonesia.

\section{Results}

The subjects in this study were 16 knee OA OA grade 2-3 patients aged 40-70 years. The subject basic characteristics show the average age was 57 years old, the average height was $1.525 \mathrm{~m}$, minimum weight was 37 $\mathrm{kg}$, maximum weight was $74 \mathrm{~kg}$ (median 62), minimum BMI was 16.015 and maximum BMI was 31.203 (median 26.399) (Table 1).

Results shows that there was a correlation between confounding factorand thequadriceps and hamstring muscle strength, which is not considered as statistically significant (Table 2). The average right quadriceps muscle strength before exercise was 12.46 and it became 18 after the exercise (Table 3).

The average pre-exercise left quadriceps muscle strength was 11.59 while the postexercise value was 17.7. The average right hamstring muscle strength before exercise was 7.29 while after practicing exercise was 7.94. The data distribution of left hamstring muscle strength before exercise was 7.5 while after practicing exercise did not show normal distributed data with a median of 7.5. The results revealed that minimum value was 5.83 and maximum value was 13.16 which did not show normal distributed data with median was 12.5 . Therefore, the minimum value was 6 and maximum value was 22.5 .

The statistical analyses applied were paired $t$ test to analyze normal distributed data and paired Wilcoxon to analyze the non-normally distributed data. The results showed that the right and left quadriceps muscle strengths improved significantly with a confidence interval (CI) of 95\% ( $\mathrm{p}=0.001)$. However, no significant improvement was found in left hamstring muscle strength.

The right quadriceps and hamstring muscle strength ratio was 1.73 before exercise and became 2.3 after the exercise (Table 4). Left quadriceps and hamstring muscle strength ratio before exercise was 1.61 and 2.26 after the exercise. Based on the paired t test statistical analysis, a significant improvement was found in quadriceps and hamstring muscle strength ratio of right and left feet.

The results show improved quadriceps and hamstring muscle strength ratio after exercise in knee OA patients. The improvement was statistically significant.

\section{Discussion}

Muscle strength of the joint is considered as one of several factors which can worsen the condition of knee OA. Muscle dysfunctions include inadequate quadriceps muscles and

Table 4 Comparison between Quadriceps and Hamstring Muscle Strength Ratio

\begin{tabular}{lcccc}
\hline & $\begin{array}{c}\text { Mean Before } \\
\text { Exercise }\end{array}$ & $\begin{array}{c}\text { Mean After } \\
\text { Exercise }\end{array}$ & t & p Value \\
\hline Right ratio & 17.399 & 2.3366 & -41.992 & 0.0008 \\
Left ratio & 1.6195 & 2.2621 & -66.116 & 0.0000 \\
\hline
\end{tabular}


quadriceps and hamstring muscle strength ratio deviation which will lead to imbalance of knee joint muscles.

This study included 16 knee OA grade 2-3 patients aged less than 70 years who visited Dr. Hasan Sadikin General Hospital and met the inclusion criteria. The subjects were given interventions in the form of quadriceps muscle strengthening isotonic exercise 24 times.

The subject average age in this study is 57.87 and the standard deviation is 2.81 . However, a previous study discovered that knee $\mathrm{OA}$ prevalence in $>45$ years old was $27.8 \%$ and $>60$ years old was $37 \%{ }^{8}$ The subjects who meet the inclusion criteria of this study relating to knee $\mathrm{OA}$ are females. Epidemiology proposes that females show higher risks suffering from OA with acute condition than males. ${ }^{8,9}$

The subject BMI median in this study is $26.39 \mathrm{~kg} / \mathrm{m}^{2}$, lower BMI category is 16.01 $\mathrm{kg} / \mathrm{m}^{2}$, and higher BMI category is $31.2 \mathrm{~kg} /$ $\mathrm{m}^{2}$. Most of the subjects in the study are categorized as overweight (66.67\%). The results show similarities to several studies which stated that majority of OA subjects are overweight. Overweight subjects show 8.1 times higher of OA risk than normal BMI. ${ }^{10-13}$

This study reveals that there are significant quadriceps muscle strength improvements. The findings are similar to the previous studies which discover significant quadriceps muscle strength improvements after performing resistance exercise with $60 \%$ intensity of $1 \mathrm{RM}$. Resistance exercise intensities performed by the subjects were $80 \%$ and $60 \%$ which show the similar muscle strength improvements and adaptation in elder population with more repetition in lower intensity. ${ }^{14-16}$ High intensity exercises for OA patients are not allowed due to higher joint compression force which cause pain in knee.

As the study included elder population with knee OA, the excercise intensity used was $60 \%$ of $1 \mathrm{RM}$. The exercise frequency was 3 times a week, at which the intensity was regarded as more effective for strengthening exercise than others. The strengthening exercise program should be done in 8 weeks to accomodate neural and muscle adaptations. ${ }^{1}$

No significant hamstring muscle strength improvement was seen after the quadriceps muscle strengthening exercise in this sudy. This finding is caused by insufficient hamstring muscle construction when the quadriceps contraction occurs.

Several studies describe electromyography (EMG) activities in hamstring muscle occured during the end of knee extension. However, this study is similar to a previous study which reveals that in open chained knee extension, hamstring muscle play a role to prevent knee anterior translation. ${ }^{18}$

Quadriceps and hamstring muscle strength ratio improvements are discovered in this study. The results show that quadriceps muscle strength significantly improve after exercise while hamstring muscle strength does not increase significantly. Therefore, the average ratio value is higher than healthy adults. ${ }^{6}$

Knee stability in sagittal plane is influenced by two muscle groups, the quadriceps and the hamstring muscle groups. Quadriceps and hamstring muscle strength imbalance will increase the risks of injury and nonnorrmal load distribution which stimulate knee OA progressivity. The therapy given to the OA patients should not only include quadriceps muscle strengthening exercise but also quadriceps and hamstring muscle strengths..$^{19,20}$

The limitation in this study is attributed to the primary research design aimed to examine the quadriceps muscle strength through quadriceps muscle strengthening exercise. Consequently, the study did not include the types and load positions as in correlation with hamstring muscles. A further study should be conducted to analyze the correlation between quadriceps and hamstring muscle strength ratio in normal population. An investigation on quadriceps and hamstring muscle strength ratio after quadriceps and hamstring muscle strengthening exercise can also be initiated to obtain accurate comparison.

\section{References}

1. Kingsbury SR, Gross HJ, Isherwood G, Conaghan PG. Osteoarthritis in Europe: impact on health status, work productivity and use of pharmacotherapies in five European countries. Rheumatology. 2014;53(5):937-47.

2. Hafez AR, Al-Johani AH, Zakaria AR, Al-Ahaideb

A, Buragadda S, Melam GR, et al. Treatment of knee osteoarthritis in relation to hamstring and quadriceps strength. J Phys Ther Sci. 2013;25(11):1401-5.

3. Segal NA, Glass NA, Torner J, Yang M, Felson DT, Sharma L, et al. Quadriceps weakness predicts 
risk for knee joint space narrowing in women in the MOST cohort. Osteoarthritis Cartilage. 2010;18(8):769-75.

4. Brandt KD, Dieppe P, Radin E. Etiopathogenesis of osteoarthritis. Med Clin North Am. 2009;93(1):1-24.

5. Palmieri-Smith RM, McLean SG, Ashton-Miller JA, Wojtys EM. Association of quadriceps and hamstrings cocontraction patterns with knee joint loading. J Athl Train. 2009;44(3):256-63.

6. Adegoke BOA, Mordi EL, Akinpenlu OA, Jaiyesimi AO. Isotonic quadriceps-hamstring strength ratios of patients with knee osteoarthritis and apparently healthy controls. Afr J Biomed Res. 2007;10(2):211-6.

7. Bennell KL, Hinman RS. A review of the clinical evidence for exercise in osteoarthritis of the hip and knee. J Sci Med Sport. 2011;14(1):4-9.

8. ZhangY,Jordan J.Epidemiology of osteoarthritis. Clin Geriatr Med. 2010;26(3):355-69.

9. Hämäläinen $S$, Solovieva $S$, Vehmas T, Luoma K, Leino-Arjas P, Hirvonen A. Genetic influences on hand osteoarthritis in Finnish women - a replication study of candidate genes. PLos One [serial on the internet]. 2014 May [cited 2016 Dec 12];9(5):[about 11p.]. Available from: http://journals.plos.org/plosone/ article?id=10.1371/journal.pone.0097417.

10. Hafez AR, Alenazi AM, Kachanathu AJ, Alroumi AM, Mohamed ES. Knee osteoarthritis: a review of literature. Phys Med Rehabil Int [serial on the internet]. 2014 May [cited 2016 Dec 12];1(5):[about 8p.]. Available from: http://austinpublishinggroup.com/ physical-medicine/fulltext/pmr-v1-id1021. php\#Introduction.

11. Blumenfeld O, Williams FMK, Hart DJ, Arden NK, Spector TD, Livshits G. Lower limbs composition and radiographic knee osteoarthritis (RKOA) in Chingford sample-A longitudinal study. Arch Gerontol Geriatr. 2012;56(1):148-54.

12. Henriksen $M$, Christensen $R$, DanneskioldSamsøe B, Bliddal H. Changes in lower extremity muscle mass and muscular strength after weight loss in obese patients with knee osteoarthritis: a prospective cohort study. Arthritis Rheum. 2012;64(2):438-42.

13. Lohmander LS, Gerhardsson de Verdier M, Rollof J, Nilsson PM, Engström G. Incidence of severe knee and hip osteoarthritis in relation to different measures of body mass: a populationbased prospective cohort study. Ann Rheum Dis. 2009;68(4):490-6.

14. Mayer F, Scharhag-Rosenberger F, Carlsohn A, Cassel M, Müller S, Scharhag J. The intensity and effects of strength training in the elderly. Dtsch Arztebl Int. 2011;108(21):359-64.

15. Aguiar GC, Do Nascimento MR, De Miranda AS, Rocha NP, Teixeira AL, Scalzo PL. Effects of an exercise therapy protocol on inflammatory markers, perception of pain, and physical performance in individuals with knee osteoarthritis. Rheumatol Int. 2015;35(3):52531.

16. Bressel E, Wing JE, Miller AI, Dolny DG. Highintensity interval training on an aquatic treadmill in adults with osteoarthritis: effect on pain, balance, function, and mobility. J Strength Cond Res. 2014;28(8):2088-96.

17. Kisner C, Colby LA. Therapeutic exercise: foundations and techniques. $6^{\text {th }}$ ed. Philadelphia: F.A. Davis; 2012.

18. Kingma I, Aalbersberg S, van Dieen JH. Are hamstrings activated to counteract shear forces during isometric knee extension efforts in healthy subjects? J Electromyogr Kinesiol. 2004;14(3):307-15.

19. Hortobágyi T, Westerkamp L, Beam S, Moody J, Garry J, Holbert D, et al. Altered hamstringquadriceps muscle balance in patients with knee osteoarthritis. Clin Biomech (Bristol, Avon). 2005;20(1):97-104.

20. Wang H, Ji Z, Jiang G, Liu W, Jiao X. Correlation among proprioception, muscular strength, and balance. J Phys Ther Sci. 2016;28(12):346872. 